\title{
Effects of Dragon Boat Training on Cytokine Production and Oxidative Stress in Breast Cancer Patients: A Pilot Study
}

\author{
Ilaria Tresoldi' ${ }^{1}$, Calogero Foti', Laura Masuelli', Giovanni Vanni Frajese ${ }^{3}$, Piero Rossi4, \\ Andrea Modesti ${ }^{1}$, Roberto Bei ${ }^{1}$, Maria Gabriella Giganti ${ }^{*}$ \\ ${ }^{1}$ Department of Clinical Sciences and Translational Medicine, University of Rome "Tor Vergata", Rome, Italy \\ ${ }^{2}$ Department of Experimental Medicine, University of Rome "Sapienza", Rome, Italy \\ ${ }^{3}$ Faculty of Motor and Adaptive Sciences, University of Cassino, Cassino, Italy \\ "Department of Experimental Medicine and Surgery, University of Rome "Tor Vergata", Rome, Italy \\ Email: "giganti@med.uniroma2.it
}

Received 30 January 2014; revised 28 February 2014; accepted 8 March 2014

Copyright (C) 2014 by authors and Scientific Research Publishing Inc.

This work is licensed under the Creative Commons Attribution International License (CC BY).

http://creativecommons.org/licenses/by/4.0/

c) (i) Open Access

\begin{abstract}
Regular exercise improves physical function and quality of life and reduces fatigue in cancer survivors; these health benefits could be due to the anti-inflammatory effects of exercise. In this study we examined the effects of a whole-body exercise programme and dragon boat paddling on the production of reactive oxygen species (ROMs), antioxidant capacity (BAP) and the circulating levels of several interleukins in breast cancer patients. Thirty four women surgically treated for breast cancer were enrolled in this study: 20 of them usually row dragon boats recreationally while 14 were sedentary. The 16-week training programme consisted of resistance and aerobic exercise, with the addition of dragon boat paddling for the last 8 weeks. Analyses of ROMs, cytokines and BAP were performed before and after 16 weeks of training. Results show a significant decrease in the ROMs value and significantly increased the BAP, IL-6, IL-8 and IL-15 levels. Exercise increased the BAP, IL-6, IL-8 and IL-15 values in the N+ patients, whereas only IL- 6 and IL-8 were higher in the N0 patients. We demonstrated that muscle-derived cytokines are released after a training program and that the resulting decreased oxidative stress conditions underline the health-benefiting effects of such activity on breast cancer patients.
\end{abstract}

\section{Keywords}

Myokines; Oxidative Stress; Breast Cancer; Exercise

\footnotetext{
${ }^{*}$ Corresponding author.

How to cite this paper: Tresoldi, I., Foti, C., Masuelli, L., Frajese, G.V., Rossi, P., Modesti, A., Bei, R. and Giganti, M.G. (2014) Effects of Dragon Boat Training on Cytokine Production and Oxidative Stress in Breast Cancer Patients: A Pilot Study. Open Journal of Immunology, 4, 22-29. http://dx.doi.org/10.4236/oji.2014.41004
} 


\section{Introduction}

Breast cancer is the most common type of cancer in women [1]. It has been reported that exercise interventions in breast cancer patients have positive effects on survival [2] [3], quality of life [4] and weight [5]; in addition, preliminary evidence suggests that exercise may also reduce the risk of cancer recurrence [6]. One of the mechanisms responsible for the health benefits of regular exercise could be the cumulative anti-inflammatory effects of repeated exercise [7] [8], which reduces oxidative stress by increasing the antioxidant capacity of the cell [9] [10], enhancing DNA repair systems and improving intracellular protein repair systems [11]. Indeed, oxidative stress is an important factor for the onset, progression and recurrence of cancer.

Regular exercise can reduce pro-inflammatory cytokines in people with chronic conditions, and this reduction relates to improvements in aerobic fitness [12]. However, whether the beneficial effects of regular physical activity in cancer survivors are due to the reduction of pro-inflammatory cytokines remains to be determined.

During and following exercise, skeletal muscle synthesises and releases a number of cytokines, named myokines, which exert their effects either systemically or locally [13]. The myokines released by skeletal muscle include interleukin (IL)-6 [14], IL-8 [15] and IL-15 [16]. IL-6 modulates the systemic inflammation response by stimulating anti-inflammatory cytokines, such as IL-1 receptor antagonist (IL-1ra), and inhibiting the production of the pro-inflammatory cytokine tumour necrosis factor (TNF)- $\alpha$ [17].

The purpose of our study was to determine the effect of 16 weeks of increased exercise training on the circulating myokines levels and oxidative stress status in breast cancer patients who usually participate in recreational dragon boat paddling. Dragon boat is a human-powered watercraft that originated in China more than 2000 years ago and is a competitive sport worldwide. Furthermore, Abreast in a Boat, conceived in Canada in 1996, was the first team of dragon boat racers comprising breast cancer patients. Dragon boat paddling is a repetitive, vigorous physical exercise that increases flexibility, aerobic capacity and strength and provides an important positive message to all people with breast cancer [18].

The results of this study might help in the understanding of the effects of sport activity on oxidative stress and muscle adaptation exercise, which induces the release of myokines in breast cancer patients.

\section{Materials and Methods}

\subsection{Participants and Training Programme}

Thirty four women surgically treated for breast cancer were enrolled in this study: 20 of them usually participate in recreational dragon boat paddling (mean age $53.45 \pm 7.75$ years) while 14 were sedentary (mean age $52.07 \pm$ 7.44 years); all the subjects were women $9 \pm 5$ years post-surgery. The exclusion criteria included cardiovascular diseases, acute somatic symptoms (e.g., fever, infection), uncontrolled hypertension, uncontrolled pain or any other condition that contraindicates exercise training in cancer survivors [19].

The TNM status could be obtained for 8 ( 3 were N0 and $5 \mathrm{~N}+$ ) and 18 (6 were N0 and $12 \mathrm{~N}+$ ) of the sedentary and dragon boat patients, respectively. The subjects enrolled in the study had no clinical or radiographic evidence of distant metastases.

It was not possible to enrol a control group consisting of healthy volunteers who participate in recreational dragon boat paddling because there is only a small number of individuals who play this sport in Italy. Indeed, this discipline is used mainly as beneficial complementary therapy for women who have had surgery for breast cancer [20], as several studies have confirmed the benefits of this physical activity on the quality of life of breast cancer patients [21] [22].

The anthropometric parameters were determined for all participants at the outset of the study. No significant differences in age, weight and height were observed when comparing the dragon boat patients exercising regularly and the sedentary patients. Conversely, the BMI was significantly higher in the dragon boat patients (p < 0.05) (data not shown). According to the Helsinki declaration, signed, informed consent was provided by all the subjects prior to entering the study. The study protocol was approved by the local ethics committee (N. $\left.{ }^{\circ} 150 / 13\right)$.

The 16-week exercise programme consisted of resistance and aerobic exercises, with the addition of dragon boat paddling in the last 8 weeks. Serum samples were collected after an overnight fast, between 9:00 a.m. and 10:00 a.m. For the dragon boat group, the first blood sample was collected before the 16 weeks of training and during the regular exercise regime, whereas the second blood sample was drawn after the increased training time. Only one sample was collected from the sedentary patients. 
The exercise programme, 2 weekly supervised sessions of 60 min. duration each, consisted of aerobic exercise and resistance training and was performed over the duration of the experimental period. In brief, each session included stretching (at the beginning and at the end), resistance exercises and aerobic effort ( 25 min. each, in the core part). The resistance training included exercises that engaged the major muscle groups (e.g., chest press, shoulder press, leg extension, leg curl, leg press, abdominal crunch, arm extension, lateral pull-down). The dragon boat training, which began at week 8 and was performed concurrently with the exercise programme for the next 8 weeks, included intermittent paddling activity in a boat once a week (60 min. duration each). The last 8 weeks of training consisted of repetitive and resistive exercise of this vigorous and intense upper-body sport. This practice was supervised by an expert investigator.

The regular exercise consisted of only a 60-min. session per week, and this exercise involved the same aerobic and resistance training described above. The dragon boat patients annually performed this regular and periodically increased exercise and boat paddling activity.

\subsection{Measurement of Cytokine Levels, Oxidative Status (ROMs) and Antioxidant Capacity (BAP)}

Commercially available human ELISA kits were employed to determine the IL-6, TNF- $\alpha$, IL-8, IL-15 and IL1ra levels (high-sensitivity Quantikine, R \& D Systems, Minneapolis, MN, USA) following the instructions of the manufacturer. The detection limits were 0.70 and $1.6 \mathrm{pg} / \mathrm{ml}$ for IL-6 and TNF- $\alpha, 3.5$ and $2.0 \mathrm{pg} / \mathrm{ml}$ for IL-8 and IL-15, respectively, and $6.26 \mathrm{pg} / \mathrm{ml}$ for IL-1ra.

The oxidative status was evaluated by measuring the serum concentrations of hydroperoxides according to an automated method by Diacron (Diacron SPF, Grosseto, Italy). This test is based on the reaction of the hydroperoxides of a biological sample with transition metals (iron), forming free radicals that then oxidise an alchilamine to produce a coloured radical detected by photometry at $505 \mathrm{~nm}$. The blood samples were mixed with an acidic buffer reagent to release iron from the plasma proteins that will react with peroxides in the blood to form free radicals; a chromogen reagent (alchilamine) is added to produce a pink-coloured derivative. This derivative is photometrically quantified, and the optical density is directly proportional to the concentration of ROMs. The references values of the d-ROMs test are between 20 - $24 \mathrm{mg} \mathrm{H}_{2} \mathrm{O}_{2} / \mathrm{dl}$, independently of gender and age. A value higher than $25.6 \mathrm{mg} \mathrm{H}_{2} \mathrm{O}_{2} / \mathrm{dl}$ indicates an increased level of oxidative stress that is linear up to $40 \mathrm{mg} \mathrm{H}_{2} \mathrm{O}_{2} / \mathrm{dl}$ [23].

The antioxidant status was examined using the biological antioxidant potential (BAP) test, according to an automated method by Diacron; the components of the antioxidant plasma barrier are directly measured by active scavengers. The blood sample is dissolved in a coloured solution that is obtained by mixing a source of ferric ions ( $\mathrm{FeCl}_{3}$, ferric chloride) with a chromogenic substrate (a thiocyanate-derived compound). After 5 min of incubation, the solution will decolourise, and the intensity of the change will be directly proportional to the ability of the plasma to reduce ferric ions. The results are expressed in units of $\mu \mathrm{mol} / \mathrm{L}$. The normal reference values of the BAP test are $>2200 \mu \mathrm{mol} / \mathrm{L}$; values below $2000 \mu \mathrm{mol} / \mathrm{L}$ indicate an antioxidant deficiency status [16] [24].

\subsection{Statistical Analysis}

The statistical analysis of the serum values was performed using SPSS 20.0 software for Windows ${ }^{\circledR}$ (SPSS, Chicago, IL, USA). The results are expressed as the mean value \pm standard deviation (SD). All the groups were analysed using the Kolmogorov-Smirnov test for a normal distribution, and significant differences between the control and experimental groups were assessed using ANOVA tests. The correlations within each group were performed using Spearman's correlation. Differences were considered significant at $\mathrm{p} \leq 0.05$.

\section{Results}

Figure 1 shows the variation in cytokine concentrations in the dragon boat group before and after increased exercise. The IL-6, IL-8 and IL-15 serum concentration significantly increased after 16 weeks of training ( $\mathrm{p}<$ 0.001), whereas the TNF- $\alpha$ values showed no significant differences.

No significant differences in the IL-6, IL-15 and TNF- $\alpha$ serum values were observed when comparing the dragon boat patients exercising regularly and the sedentary patients. Conversely, the IL-8 concentration was sig- 
nificantly higher in the sedentary patients $(\mathrm{p}<0.05)$ (data not shown).

The IL-1ra value increased after 16 weeks of training but was not significant; similar to what was observed for IL-8, the values in the sedentary patients were higher than the dragon boat patients with regular exercise $(\mathrm{p}<$ 0.05) (data not shown).

The ROMs values decreased after training, though this decrease was not significant. In addition, the ROMs values were higher in the sedentary patients than in the group with regular exercise $(\mathrm{p}<0.05)$ (Figure 2(a)).

However, the ROMs values returned to the normal range after increased training yet remained above the range during regular exercise and in the sedentary patients.

BAP significantly increased after 16 weeks of training $(\mathrm{p}<0.001)$. No significant differences were found between the sedentary patients and dragon boat patients before the increase in the exercise regime (Figure 2(b)).

Overall, an inverse and significant correlation between ROMs and BAP both before and after increased exercise was observed in the dragon boat group. Conversely, no correlation was found between ROMs and BAP in the sedentary patients (Table 1).

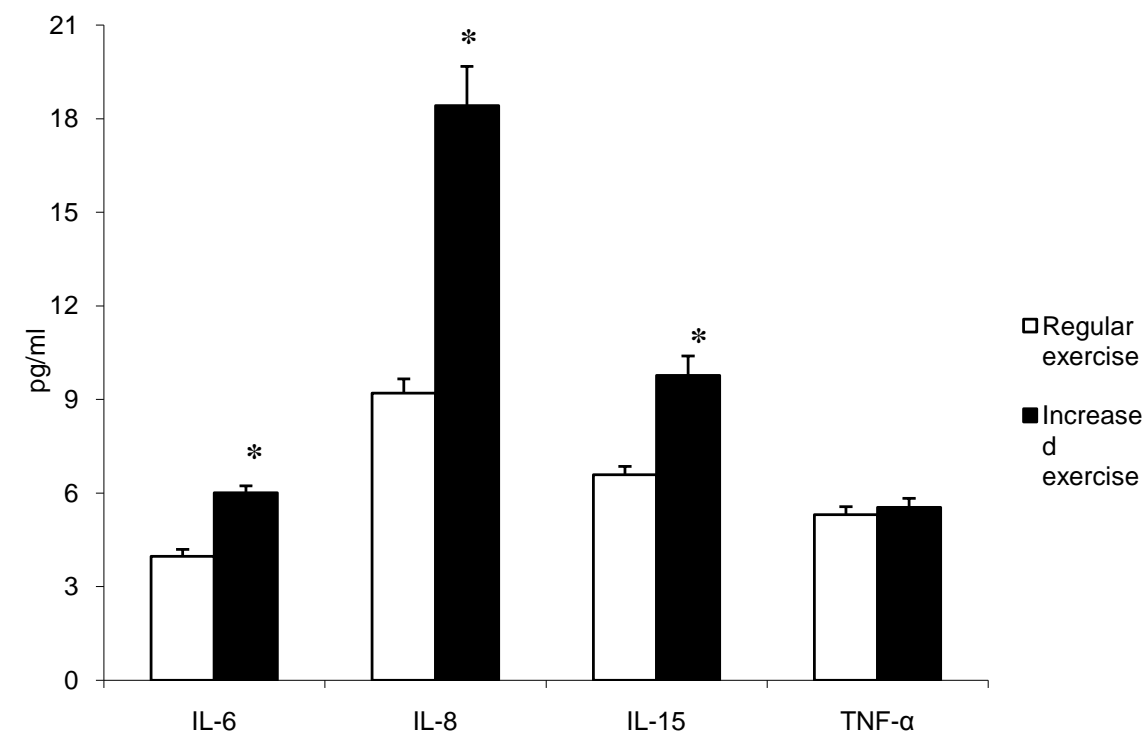

Figure 1. Serum levels of IL-6, IL-8, IL-15 and TNF- $\alpha$. IL-6, IL-8 and IL-15 were significantly increased in the dragon boat patients after 16 weeks of training ( ${ }^{*}<0.001$ ); the TNF- $\alpha$ values show no significant differences.

ROMs

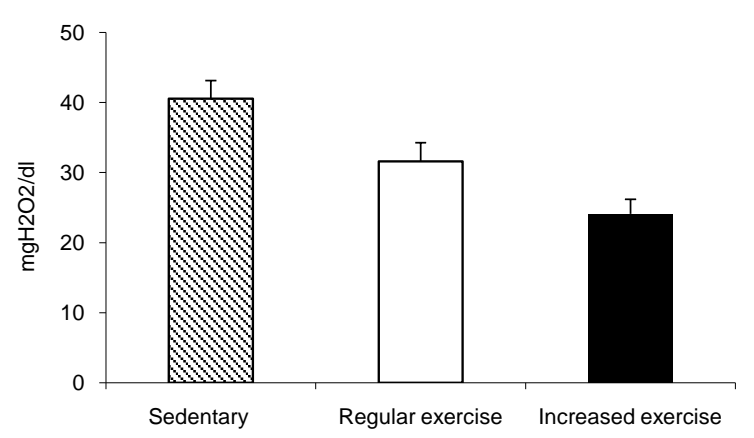

(a)

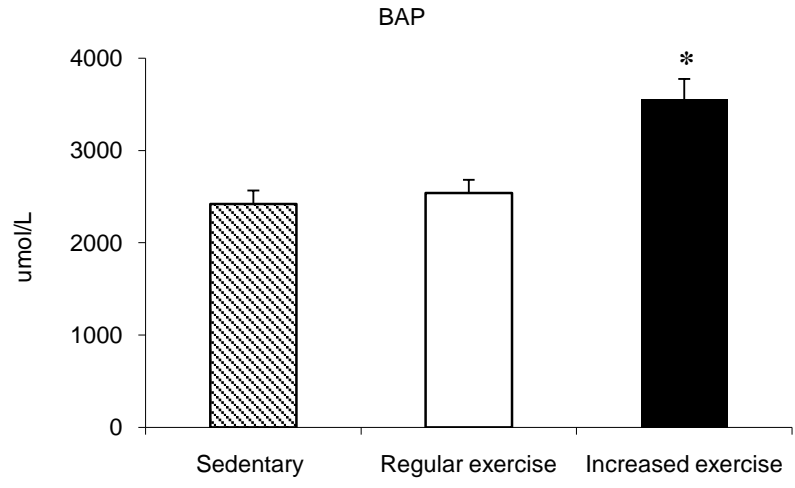

(b)

Figure 2. Reactive oxygen metabolites (ROMs) and biological antioxidant potential (BAP), (a) the ROMs values decreased after 16 weeks of training in the dragon boat patients, but this decrease was not significant; the values were higher in the sedentary patients than in the dragon boat patients with regular exercise $(\bullet p<0.05)$, (b) the BAP values of the dragon boat patients were significantly higher after 16 weeks of training ( $\left.{ }^{*} \mathrm{p}<0.001\right)$. No significant differences were found between the sedentary patients and dragon boat patients with regular exercise. 
An effect of the training programme was observed when comparing the $\mathrm{N} 0$ and $\mathrm{N}+$ subgroups of the dragon boat patients. Indeed, IL-6 and IL-8 were enhanced $(\mathrm{p}<0.05)$ in the N0 patients after increased training. In addition, the levels of IL-6 and IL-8 ( $>0.001)$ and IL-15 and BAP $(\mathrm{p}<0.05)$ were higher in the N+ dragon boat patients after 16 weeks of increased exercise (Table 2).

No significant differences were found in the $\mathrm{N}+$ patients when comparing the results obtained for the sedentary patients versus the dragon boat patients with regular exercise. In contrast, the ROMs values were significantly higher in the N0 sedentary patients (data not shown).

\section{Discussion}

There is evidence to support that regular exercise improves physical function [25], quality of life and fatigue in cancer survivors, particularly in breast cancer [26] [27]. Our results indicate a positive influence of physical exercise, which reduces the oxidative status and increases the antioxidant capacity. Indeed, the benefits of exercise were emphasised by the lower ROMs value in the active patients. Radak et al. [8] attributed the increase in antioxidant capacity after exercise to the increased formation of superoxide dismutase and glutathione peroxidase; the long-term effects of exercise-induced oxidative stress amelioration are reflected in the induction of adaptation [28], particularly in the increase of antioxidant enzyme activity [29] [30].

Currently, there is an ongoing debate with regard to the influence of physical activity on cancer. The present study provides evidence that dragon boat training positively influences the oxidative stress condition in breast cancer patients.

The possibility of assessing the effects of regular exercise on cytokine levels in breast cancer patients is a question of important medical relevance because it provides a prognostic significance in this disease [31] [32]. During exercise, the working skeletal muscles release anti-inflammatory cytokines, such as IL-6, significantly attenuating several symptoms, including a poor quality of life or fatigue. The present data suggest that the documented benefits of regular exercise in cancer patients could be mediated by the changes in cytokine concentrations, as observed in our training group. IL-6 has been classified as both a pro- and anti-inflammatory cytokine [33] [34], but the recent opinion is that IL-6 possesses primarily anti-inflammatory effects. Furthermore, IL-6 directly inhibits TNF- $\alpha$ and IL-1 expression and is an inducer of IL-1ra, which exerts anti-inflammatory activity by blocking the IL-1 receptor [35]. The increase in IL-1ra in response to exercise could have reduced the biological activity of pro-inflammatory IL-1. The exercise-induced cytokine cascade causes an increase in IL-6 and IL-1ra [36], creating an anti-inflammatory environment that inhibits TNF- $\alpha$ release.

IL-8 and IL-15, together with IL-6, are exercise-induced cytokines [37].

IL-8 is a well-known chemokine that attracts neutrophils and is regulated by muscle contraction [18]. IL-15 is expressed in human skeletal muscle and has been identified as an anabolic factor in muscle growth; it also appears to play a role in lipid metabolism. The data obtained in our study showed an increase in IL-8 and IL-15 concentrations after 16 weeks of increased training, a period that was characterised by repetitive and intense exercise.

Taken together, myokines may be involved in mediating the anti-inflammatory effects of exercise.

Table 1. Correlation between oxidative status (ROMs) and antioxidant capacity (BAP) in dragon boat patients, during regular exercise and after increased exercise, and Sedentary patients ( $r=$ correlation coefficient).

\begin{tabular}{cccc} 
& & BAP & \\
\cline { 2 - 4 } & Regular exercise & Increased exercise & Sedentary \\
\hline ROMs & $r=-0.578 \mathrm{p}<0.008$ & $r=-0.657 \mathrm{p}<0.002$ & $r=0.182 \mathrm{NS}$ \\
\hline
\end{tabular}

Table 2. Statistical differences observed in N0 and N+ dragon boat patients comparing regular exercise versus increased exercise.

\begin{tabular}{ccccccccc}
\hline Dragon boat patients & & IL-8 & IL-1ra & IL-15 & IL-6 & TNF- $\alpha$ & ROMs & BAP \\
\hline $\begin{array}{c}\text { Regular exercise vs } \\
\text { Increased exercise }\end{array}$ & N0 & $\mathrm{p}<0.05$ & NS & NS & $\mathrm{p}<0.05$ & NS & NS & NS \\
\hline
\end{tabular}


The influence of exercise was manifested in both $\mathrm{N} 0$ and $\mathrm{N}+$ patients: we observed an increase in myokines and antioxidant capacity with increased training time, whereas the ROMs values decreased. We did not, however, observe any significant differences in $\mathrm{N} 0$ versus $\mathrm{N}+$ in the dragon boat patients with regular exercise or in the dragon boat patients after increased exercise.

A larger sample size, with different nodal staging, needs to be evaluated to reach a definite conclusion regarding the different cytokine levels found in the N0 and $\mathrm{N}+$ patients.

\section{Conclusions}

There is evidence from intervention studies to support that regular exercise improves physical function, quality of life and fatigue in cancer survivors [24] [29]. The purpose of our study was to investigate the effect of 16 weeks of training (aerobic-resistance+paddling) on circulating cytokines and oxidative stress in breast cancer patients who participate in dragon boat paddling. This ancient sport has been assessed for its psychosocial benefits, and it was shown to have positive effects on lymphedema in women with breast cancer [17].

Overall, a combined 16-week exercise training programme (aerobic-resistance+dragon boat) induced changes in myokines levels in breast cancer patients; these myokines provide the cross-talk between the immune system and muscle cells [38] [39]. Furthermore, exercise may reduce inflammatory oxidative damage by increasing the antioxidant capacity and reducing the oxidative status.

Our study confirms that skeletal muscle may be considered as an endocrine organ that produces and releases myokines, and the health-beneficial effects of exercise were emphasised by a decrease in the oxidative status. These observed improvements were due to exercise training and dragon boat paddling.

\section{Acknowledgements}

The authors thank Susan G. Komen Italy Onlus for the financial support and the Pink Butterfly dragon boat team, Rome (Italy).

\section{Conflict of Interest}

The authors declare that there is no conflict of interests regarding the publication of this article.

\section{References}

[1] American Cancer Society (2011) Cancer statistics presentation. American Cancer Society, Inc. (2011) 1-60.

[2] Holmes, M.D., Chen, W.Y., Feskanich, D., Kroenke, C.H. and Colditz, G.A. (2005) Physical Activity and Survival after Breast Cancer Diagnosis. JAMA, 293, 2479-2486. http://dx.doi.org/10.1001/jama.293.20.2479

[3] Irwin, M.L., Smith, A.W., McTiernan, A., Ballard-Barbash, R., Cronin, K., Gilliland, F.D., Baumgartner, R.N., Baumgartner, K.B. and Bernstein, L. (2008) Influence of Pre- and Postdiagnosis Physical Activity on Mortality in Breast Cancer Survivors: The Health, Eating, Activity, and Lifestyle Study. Journal of Clinical Oncology, 26, 3958-3964. http://dx.doi.org/10.1200/JCO.2007.15.9822

[4] Speed-Andrews, A.E. and Courneya, K.S. (2009) Effects of Exercise on Quality of Life and Prognosis in Cancer Survivors. Current Sports Medicine Reports, 8, 176-181. http://dx.doi.org/10.1249/JSR.0b013e3181ae98f3

[5] Ligibel, J.A., Campbell, N., Partridge, A., Chen, W.Y., Salinardi, T., Chen, H., Adloff, K., Keshaviah, A. and Winer, E.P. (2008) Impact of a Mixed Strength and Endurance Exercise Intervention on Insulin Levels in Breast Cancer Survivors. Journal of Clinical Oncology, 26, 907-912. http://dx.doi.org/10.1200/JCO.2007.12.7357

[6] Speck, R.M., Courneya, K.S., Mâsse, L.C., Duva,l S. and Schmitz, K.H. (2010) An Update of Controlled Physical Activity Trials in Cancer Survivors: A Systematic Review and Meta-Analysis. Journal of Cancer Survivorship, 4, 87-100. http://dx.doi.org/10.1007/s11764-009-0110-5

[7] Pinto, A., Di Raimondo, D., Tuttolomondo, A., Buttà, C., Milio, G. and Licata, G. (2012) Effects of Physical Exercise on Inflammatory Markers of Atherosclerosis. Current Pharmaceutical Design, 18, 432643-432649. http://dx.doi.org/10.2174/138161212802481192

[8] Crisafulli, A., Tocco, F., Melis, F., Milia, R., Concu A. (2013) Natural Killer Cells Responsiveness to Physical Exercise: A Brief Review. Open Journal of Immunology, 3, 190-200. http://dx.doi.org/10.4236/oji.2013.34024

[9] Radak, Z., Chung, H.Y. and Goto, S. (2008) Systemic Adaptation to Oxidative Challenge Induced by Regular Exercise. Free Radical Biology \& Medicine, 44,153-159. http://dx.doi.org/10.1016/j.freeradbiomed.2007.01.029

[10] Grosso, G., Bei, R., Mistretta, A., Marventano, S., Calabrese, G., Masuelli, L., Giganti, M.G., Modesti, A., Galvano, F. 
and Gazzolo, D. (2013) Effects of Vitamin C on Health: A Review of Evidence. Frontiers in Bioscience (Landmark ed), 1, 1017-1029.

[11] Rogers, C.J., Colbert, L.H., Greiner, J.W., Perkins, S.N. and Hursting, S.D. (2008) Physical Activity and Cancer Prevention: Pathways and Targets for Intervention. Sports Medicine, 38, 271-296. http://dx.doi.org/10.2165/00007256-200838040-00002

[12] Goldhammer, E., Tanchilevitch, A., Maor, I., Beniamini, Y., Rosenschein, U. and Sagiv, M. (2005) Exercise Training Modulates Cytokines Activity in Coronary Heart Disease Patients. International Journal of Cardiology, 100, 93-99. http://dx.doi.org/10.1016/j.ijcard.2004.08.073

[13] Pedersen, B.K., Akerstrom, T.C., Nielsen, A.R. and Fischer, C.P. (2007) Role of Myokines in Exercise and Metabolism. Journal of Applied Physiology, 103, 1093-1098. http://dx.doi.org/10.1152/japplphysiol.00080.2007

[14] Steensberg .A., van Hall, G., Osada, T., Sacchetti, M., Saltin, B. and Pedersen, B.K. (2000) Production of Interleukin-6 in Contracting Human Skeletal Muscles Can Account for the Exercise Induced Increase in Plasma Interleukin-6. The Journal of Physiology, 529, 237-242. http://dx.doi.org/10.1111/j.1469-7793.2000.00237.x

[15] Akerstrom, T., Steensberg, A., Keller, P., Keller, C., Penkowa, M. and Pedersen, B.K. (2005). Exercise Induces Interleukin-8 Expression in Human Skeletal Muscle. The Journal of Physiology, 563, 507-516. http://dx.doi.org/10.1113/jphysiol.2004.077610

[16] Nielsen, A.R., Mounier, R., Plomgaard, P., Mortensen, O.H., Penkowa, M., Speerschneider, T., Pilegaard, H. and Pedersen, B.K. (2007) Expression of Interleukin-15 in Human Skeletal Muscle-Effect of Exercise and Muscle Fibre Type Composition. The Journal of Physiology, 584, 305-312. http://dx.doi.org/10.1113/jphysiol.2007.139618

[17] Petersen, A.M. and Pedersen, B.K. (2005) The Anti-Inflammatory Effect of Exercise. Journal of Applied Physiology, 98, 1154-1162. http://dx.doi.org/10.1152/japplphysiol.00164.2004

[18] Harris, S.R. (2012) We're All in the Same Boat: A Review of the Benefits of Dragon Boat Racing for the Women Living with Breast Cancer. Evidence-Based Complementary and Alternative Medicine, Article ID: 167651. http://dx.doi.org/10.1155/2012/167651

[19] Lucia, A., Earnest, C. and Perez, M. (2003) Changed-Related Fatigue: Can Exercise Physiology Assist Oncologys? The Lancet Oncology, 4, 616-625. http://dx.doi.org/10.1016/S1470-2045(03)01221-X

[20] Lane, K., Jespersen, D. and McKenzie, D.C. (2005) The effect of a Whole Body Exercise Programme and Dragon Boat Training on Arm Volume and Arm Circumference in Women Treated for Breast Cancer. European Journal of Cancer Care, 14, 353-358. http://dx.doi.org/10.1111/j.1365-2354.2005.00595.x

[21] McNeely, M.L., Campbell, K.L., Courneya, K.S. and Mackey, J.R. (2009) Effect of Acute Exercise on Upper-Limb Volume in Breast Cancer Survivors: A Pilot Study. Physiotherapy Canada, 61, 242-251. http://dx.doi.org/10.3138/physio.61.4.244

[22] Warburton, D.E., Sheel, A.W., Hodges, N., Stewart, I.B., Yoshida, E.M., Levy, R.D. and McKenzie, D.C. (2004) Effects of Upper Extremity Exercise Training on Peak Aerobic and Anaerobic Fitness in Patients after Transplantation. American Journal of Cardiology, 93, 939-943. http://dx.doi.org/10.1016/j.amjcard.2003.12.030

[23] Simiakakis, M., Kapsimalis, F., Chaligiannis, E., Loukides, S., Sitaras, N. and Alchanatis, M. (2012) Lack of Effect of Sleep Apnea on Oxidative Stress in Obstructive Sleep Apnea Syndrome (OSAS) Patients. PLoS ONE, 7, Article ID: e39172. http://dx.doi.org/10.1371/journal.pone.0039172

[24] Benzie, F. and Strain, J.J. (1996) The Ferric Reducing Ability of Plasma (FRAP) as a Measure of “Antioxidant Power”: The FRAP Assay. Analytical Biochemistry, 239, 70-76. http://dx.doi.org/10.1006/abio.1996.0292

[25] Yamaguchi, N., Chen, R., Okamoto, K., Takei, T., Tsubokawa, M., Sakamoto, D., Jia, X., Wan, W. and Murayama, T. (2013) Quantitative Regulation of Peripheral Leukocyte by Light Exercise and Tailored Scale for Assessment. Open Journal of Immunology, 3, 175-183.

[26] Irwin, M.L. (2009) Physical Activity Interventions for Cancer Survivors. British Journal of Sports Medicine, 43, 32-38. http://dx.doi.org/10.1136/bjsm.2008.053843

[27] Winters-Stone, K.M., Schwartz, A. and Nail, L.M. (2010) A Review of Exercise Interventions to Improve Bone Health in Adult Cancer Survivors. Journal of Cancer Survivorship, 4, 187-201. http://dx.doi.org/10.1007/s11764-010-0122-1

[28] McArdle, A. and Jackson, M.J. (2000) Exercise, Oxidative Stress and Ageing. Journal of Anatomy, 197, 539-541. http://dx.doi.org/10.1046/j.1469-7580.2000.19740539.x

[29] Duncan, K., Harris, S. and Ardies, C.M. (1997) Running Exercise May Reduce Risk for Lung and Liver Cancer by Inducing Activity of Antioxidant and Phase II Enzymes. Cancer Letters, 116, 151-159. http://dx.doi.org/10.1016/S0304-3835(97)00189-4

[30] Nakatani, K., Komatsu, M., Kato, T., Yamanaka, T., Takekura, H., Wagatsuma, A., Aoyama, K., Xu, B., Hirano, T., Kasai, H., Ando, S. and Takeuchi, T. (2005) Habitual Exercise Induced Resistance to Oxidative Stress. Free Radical Research, 39, 905-911. http://dx.doi.org/10.1080/10715760500183300 
[31] Seruga, B., Zhang, H., Berstein, L.J. and Tannock, J.F. (2008) Cytokines and Their Relationship to the Symptons and Outcome of Cancer. Nature Reviews Cancer, 8, 887-899. http://dx.doi.org/10.1038/nrc2507

[32] Bei, R., Guptill, V., Masuelli, L., Kashmiri, S.V., Muraro, R., Frati, L., Schlom, J. and Kantor, J. (1998) The Use of a Cationic Liposome Formulation (DOTAP) Mixed with a Recombinant Tumor-Associated Antigen to Induce Immune Responses and Protective Immunity in Mice. Journal of Immunotherapy, 21, 159-169. http://dx.doi.org/10.1097/00002371-199805000-00001

[33] Giganti, M.G., Liuni, F., Celi, M., Gasbarra, E., Zenobi, R., Tresoldi, I., Modesti, A., Bei, R. and Tarantino, U. (2012) Changes in Serum Levels of TNF- $\alpha$, IL-6, OPG, RANKL and Their Correlation with Radiographic and Clinical Assessment in Fragility Fractures and High Energy Fractures. Journal of Biological Regulators \& Homeostatic Agents, 26, 671-680.

[34] Signore, A., Chianelli, M., Bei, R., Oyen, W. and Modesti, A. (2003) Targeting Cytokine/Chemokine Receptors: A Challenge for Molecular Nuclear Medicine. European Journal of Nuclear Medicine and Molecular Imaging, 30, 149-156. http://dx.doi.org/10.1007/s00259-003-1149-6

[35] Barton, B.E. (1997) IL-6 Insights into Novel Biological Activities. Clinical Immunology and Immunophatology, 85, 16-20. http://dx.doi.org/10.1006/clin.1997.4420

[36] Ostrowski, K., Schjerling, P. and Pedersen, B.K. (2000) Physical Activity and Plasma IL-6 Levels in Humans: Effect of Intensity of Exercise. European Journal of Applied Physiology, 83, 512-515. http://dx.doi.org/10.1007/s004210000312

[37] Nielsen, A.R. and Pedersen, B.K. (2007) The Biological Roles of Exercise-Induced Cytokines: IL-6, IL-8, and IL-15. Applied Physiology, Nutrition, and Metabolism, 32, 833-839. http://dx.doi.org/10.1139/H07-054

[38] Pedersen, B.K. (2009) The Diseasome of Physical Inactivity and the Role of Myokines in Muscle-Fat Cross-Talk. Journal of Physiology, 587, 5559-5568. http://dx.doi.org/10.1113/jphysiol.2009.179515

[39] Ertek, S. and Cicero, A. (2012) Impact of Physical Activity on Inflammation: Effects on Cardiovascular Disease Risk and Other Inflammatory Conditions. Archives of Medical Science, 8, 794-804. http://dx.doi.org/10.5114/aoms.2012.31614 